Document downloaded from:

http://hdl.handle.net/10251/147535

This paper must be cited as:

Lan, Y.; Peris Manguillot, A. (2018). Weak stability of non-autonomous discrete dynamical systems. Topology and its Applications. 250:53-60.

https://doi.org/10.1016/j.topol.2018.10.006

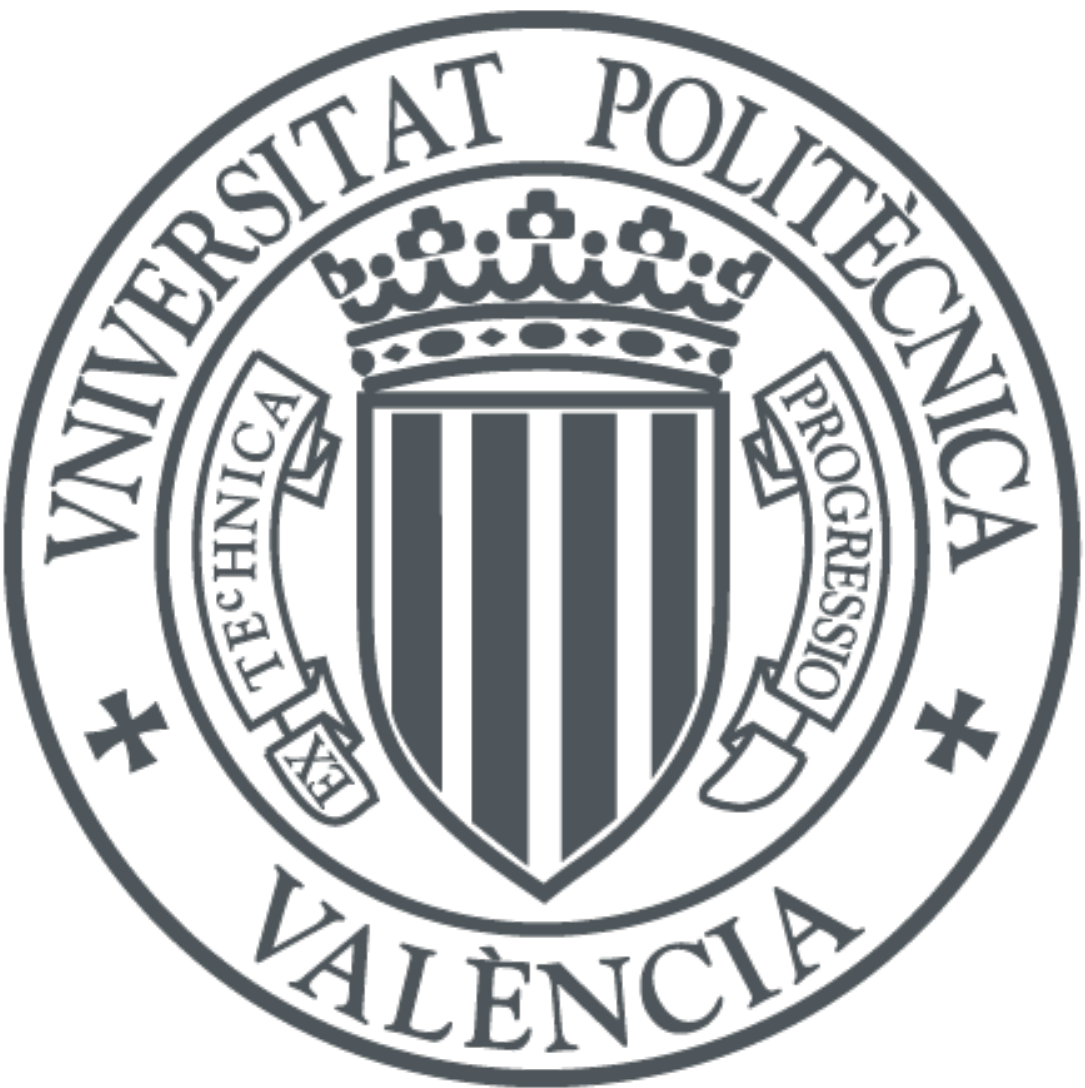

The final publication is available at

https://doi.org/10.1016/j.topol.2018.10.006

Copyright Elsevier

Additional Information 


\title{
Weak stability of non-autonomous discrete dynamical systems
}

\author{
Yaoyao Lan ${ }^{1,}$, Alfred Peris ${ }^{2}$ \\ ${ }^{a}$ Department of Mathematics \\ Chongqing University of Arts and Sciences, 402160 Yongchuan, China \\ ${ }^{b}$ Institut Universitari de Matemtica Pura $i$ Aplicada \\ Universitat Politècnica de València, Edifici 8E, Acces F, 4a planta, 46022 València, \\ Spain
}

\begin{abstract}
In this paper we introduce a concept of weak stability in non-autonomous dynamical system. We show that the set of weak stable points is residual and investigate the relation between weak stability and shadowing property. We also discuss the relation between weak stability of non-autonomous dynamical system and its induced set-valued system.
\end{abstract}

Keywords: weak stability, non-autonomous dynamical system, set-valued system, shadowing property

\section{Introduction}

Let $f: X \rightarrow X$ be a continuous map acting on a compact metric space $(X, d)$. A autonomous discrete dynamical system is a pair $(X, f)$. A nonautonomous discrete system difference equation is the following:

$$
x_{n+1}=f_{n}\left(x_{n}\right), \quad n \geq 0,
$$

where $\left\{f_{n}\right\}_{n=0}^{\infty}$ is a sequence of continuous maps and each $f_{n}$ is a self-map on $X$. Set $F=\left\{f_{n}\right\}_{n=0}^{\infty}$ for the sake of simplicity. Note that the autonomous dynamical system is a special case of system (1) when $f_{n}=f$ for all $n \geq 0$.

We refer to Section 2 for other notions and notations mentioned in this section.

Email addresses: yylan@cqwu.edu.cn (Yaoyao Lan), aperis@mat.upv.es (Alfred Peris $\left.^{2}\right)$ 
Non-autonomous dynamical systems deal with the situations which dynamics can vary with time. Recently, the study of non-autonomous dynamical systems become active and many elegant results have been obtained $[1,2,3,4,5,6]$. The dynamics in non-autonomous case can be vary complicated. Hence it is natural to study the pseudo-orbits for a better understanding of true orbits. Along this line, the study of shadowing property in autonomous dynamical systems attracts lots of attention $[7,8,10,11,12,13,14$, and the references therein]. In [9], a concept of weak stability has been introduced, and it is shown that orbital shadowing property is generic in the set of weak stable homeomorphisms. Motivated by this idea, we discuss weak stability in nonautonomous dynamical systems.

On the other hand, a discrete dynamical system uniquely induces its setvalued system which on the space of compact subsets. It is natural to ask the following question: What is the relation between dynamical properties of the original and set-valued systems? The study of the dynamics of the induced system has been extensively studied and many elegant results have been obtained[15, 16, 17, and the references therein].

In this present paper, a concept of weak stability has been introduced and the relation between shadowing and weak stability has been discussed. The relations between some chaotic properties of the nonautonomous discrete dynamical system and its set-valued system have also been investigted.

Below, basic notions are introduced in Section 2. Main results are presented in Section 3.

\section{Basic concepts and notations}

Let $F=\left\{f_{n}\right\}_{n=0}^{\infty}$ be a sequence of continuous selfmaps defined on a compact metric space $X$. An orbit of a point $x_{0} \in X$, denoted by $o(x, F)=$ $\left\{x_{n}\right\}_{n=0}^{\infty}$, is defined as follows:

$$
x_{n}=f_{n}\left(x_{n-1}\right), \quad n=1,2, \cdots
$$

3 Denote $F_{n}: X \rightarrow X$ by

$$
F_{n}=f_{n} \circ f_{n-1} \cdots \circ f_{2} \circ f_{1} .
$$

For $\delta>0$, a $\delta$-pseudo-orbit for $F$ is a sequence $\left\{x_{n}\right\}_{n=0}^{\infty}$ in $X$ such that $d\left(f_{i+1}\left(x_{i}\right), x_{i+1}\right)<\delta$ for $i \in \mathbb{N}$. A finite $\delta$-pseudo-orbit $\left\{x_{i}\right\}_{i=0}^{b}$ is called a $\delta$-chain from $x_{0}$ to $x_{b}$ with length $b+1$. 
For $\epsilon>0, F$ has shadowing property if, there is a $\delta>0$ such that every $\delta$-pseudo-orbit for $F$ can be $\epsilon$-shadowed by some point $y \in X$, that is $d\left(F_{i}(y), x_{i}\right)<\delta$ for all $i \in \mathbb{N}$, where $\mathbb{N}$ denotes the set of all positive integers. $F$ is chain transitive if for any $x, y \in X$ there is a $\delta$-chain of $F$ from $x$ to $y$.

Let $\mathcal{K}(X)$ be the collection of all non-empty compact subsets of $X$. Define the $\varepsilon$-neighborhood of a nonempty subset $A$ in $X$ to be the set

$$
\mathrm{N}_{\varepsilon}(A)=\{x \mid d(x, A)<\varepsilon\},
$$

where $d(x, A)=\inf _{a \in A} \rho(x-a)$.

The Hausdorff separation $\rho(A, B)$ of $A, B \in \mathcal{K}(X)$ is defined by

$$
\rho(A, B)=\inf \left\{\varepsilon>0 \mid A \subseteq \mathrm{N}_{\varepsilon}(B)\right\},
$$

The Hausdorff metric on $\mathcal{K}(X)$ is defined by letting

$$
H_{d}(A, B)=\max \{\rho(A, B), \rho(B, A)\} .
$$

For a compact space $X$, the topology generated by $H_{d}$ coincides with the finite topology. In this case $\mathcal{K}_{\mathcal{F}}(X)$, the set of all finite subsets of $X$ is dense in $\mathcal{K}(X)$. Also, $\mathcal{K}(X)$ is compact if and only if $X$ is compact.

\section{Main Results}

In this section, we investigate the so-called weak stability in $(X, F)$ (recall that $\left.F=\left\{f_{n}\right\}_{n=0}^{\infty}\right)$.

Definition 3.1. We call $x$ a weak stable point of $F$, or $F$ is weak stable at $x$, if for every $\epsilon>0$ there exist $\delta>0$ and an integer $T$ such that $o(z, F) \subset$ $\mathrm{N}_{\epsilon}\left(\left\{F_{i}(z) ; i=-T, \ldots, T\right\}\right)$ for any $z \in X$ with $d(z, x)<\delta$.

Theorem 3.2. Let $\left\{f_{n}\right\}_{n=0}^{\infty}$ be a sequence of homeomorphisms on a compact space $X$. Then the set of weak stable points is residual in $X$.

Proof. Let $\epsilon>0$ and $U=\left\{U_{i} \mid i=1,2, \cdots, k\right\}$ be a finite open covering of $X$ with $\operatorname{diam}\left(U_{i}\right)<\frac{\epsilon}{2}$. Set $\mathrm{K}=\{1,2, \cdots, k\}$. For every $x \in X$, choose $L_{x} \subset \mathrm{K}$ satisfying the following:
1. $o(x, F) \subset \cup\left\{U_{i} \mid i \in L_{x}\right\}$
2. $o(x, F) \cap U_{i} \neq \emptyset$ 
83

Let $W_{\epsilon}$ be the set of all $a \in X$ such that for $\epsilon>0$, there exist $\delta_{a}>0$ and positive integer $T_{a}$ with $d(a, x)<\delta_{a}$ implies $o(x, F) \subset \mathrm{N}_{\epsilon}\left(\left\{F_{i}(x)\right\}\right)$ for $i=-T_{a}, \cdots, T_{a}$. Obviously, $W_{\epsilon}$ is open. To prove that $W_{\epsilon}$ is dense in $X$, fix any $a \in X$. Choose $\lambda_{1}>0$ such that for every $x \in \mathrm{N}_{\lambda_{1}}(a)$,

$$
d\left(F_{i}(a), F_{i}(x)\right)<\frac{\epsilon}{2}
$$

where $i=-T_{a}, \cdots, T_{a}$.

Assume that $a \notin W_{\epsilon}$. For $0<\delta_{1}<\lambda_{1}$ there exists $a_{1} \in \mathrm{N}_{\delta_{1}}(a)$ such that for $i=-T_{a}, \cdots, T_{a}$,

$$
d\left(F_{m_{1}}\left(a_{1}\right), F_{i}\left(a_{1}\right)\right) \geq \epsilon,
$$

where $\left|m_{1}\right|>T_{a}$. We also have for $i=-T_{a}, \cdots, T_{a}$,

$$
d\left(F_{m_{1}}\left(a_{1}\right), F_{i}(a)\right) \geq \frac{\epsilon}{2},
$$

Indeed, if $d\left(F_{m_{1}}\left(a_{1}\right), F_{i}(a)\right)<\frac{\epsilon}{2}$, then

$$
d\left(F_{m_{1}}\left(a_{1}\right), F_{i}\left(a_{1}\right)\right) \leq d\left(F_{m_{1}}\left(a_{1}\right), F_{i}(a)\right)+d\left(F_{i}(a), F_{i}\left(a_{1}\right)\right)<\frac{\epsilon}{2}+\frac{\epsilon}{2}=\epsilon,
$$

which is a contradiction. Consequently,

$$
F_{m_{1}}\left(a_{1}\right) \notin \mathrm{N}_{\frac{\epsilon}{2}}\left(\left\{F_{i}(a)\right\}_{i=-T_{a}}^{T_{a}}\right) .
$$

Notice that $\operatorname{diam}\left(U_{i}\right)<\frac{\epsilon}{2}$, thus $F_{m_{1}}\left(a_{1}\right) \notin U_{i}$ for all $i \in L_{a}$, and then there exists $j \in \mathrm{K}-L_{a}$ such that $F_{m_{1}}\left(a_{1}\right) \in U_{j}$. Thus $L_{a} \subset L_{a_{1}}$. Choose a positive integer $m_{2}>m_{1}$ such that for all $j \in L_{a_{1}}$,

$$
\left\{F_{i}\left(a_{1}\right)\right\}_{i=-m_{2}}^{m_{2}} \cap U_{j} \neq \emptyset \text {. }
$$

Thus

$$
o\left(a_{1}, F\right) \subset \mathrm{N}_{\epsilon}\left(\left\{F_{i}\left(a_{1}\right)\right\}_{i=-m_{2}}^{m_{2}}\right) .
$$

so Still, one could choose $\lambda_{2}>0$ such that for every $x \in \mathrm{N}_{\lambda_{2}}\left(a_{1}\right)$,

$$
d\left(F_{i}\left(a_{1}\right), F_{i}(x)\right)<\frac{\epsilon}{2} .
$$

where $i=-m_{2}, \cdots, m_{2}$.

If $a_{1} \in W_{\epsilon}$ then the proof is done, otherwise there exists $a_{2} \in \mathrm{N}_{\delta_{2}}\left(a_{1}\right) \subset$ $\mathrm{N}_{\delta_{1}}(a)$ implies for $i=-m_{2}, \cdots, m_{2}$,

$$
d\left(F_{m_{3}}\left(a_{2}\right), F_{i}\left(a_{2}\right)\right) \geq \epsilon
$$


where $\left|m_{3}\right|>m_{2}$.

Using the same technique as above we obtain

$$
F_{m_{3}}\left(a_{2}\right) \notin \mathrm{N}_{\frac{\epsilon}{2}}\left(\left\{F_{i}\left(a_{1}\right)\right\}_{i=-m_{2}}^{m_{2}}\right),
$$

and then $F_{m_{3}}\left(a_{2}\right) \notin U_{i}$ for all $i \in L_{a_{1}}$, hence there exists $j \in \mathrm{K}-L_{a_{1}}$ such that $F_{m_{3}}\left(a_{2}\right) \in U_{j}$. Consequently, $L_{a_{1}} \subset L_{a_{2}}$.

By continuing this process there is $a^{*} \in \mathrm{N}_{\delta_{1}}(a)$ such that $L_{a^{*}}=\mathrm{K}$, since $\mathrm{K}$ is finite. Thus $a^{*} \in W_{\epsilon}$, which completes the proof of density of the set $W_{\epsilon}$. Set $W=\cap_{n=1}^{\infty} W_{\frac{1}{n}}$, then $W$ is residual in $X$.

Lemma 3.3. If $F$ has the shadowing property, then so does $F_{k}$ for $k \in \mathbb{N}$.

Lemma 3.4. Let $F_{k}$ be chain transitive for $k \in \mathbb{N}$. If $F$ has the shadowing property, then $F_{k}$ is topological transitive.

Proof. By Lemma 3.3, $F_{k}$ has the shadowing property. Let $\mathrm{B}\left(x, r_{1}\right)$ and $\mathrm{B}\left(y, r_{2}\right)$ be balls of $x, y \in X$, respectively. For $0<\epsilon<\min \left\{r_{1}, r_{2}\right\}$, there exists $\delta>0$ such that every $\delta$-pseudo-orbit of $F_{k}$ can be $\epsilon$-shadowed by some point of $X$. Since $F_{k}$ is chain transitive, there exists a $\delta$-chain $\{x=$ $\left.x_{0}, \cdots, x_{n}=y\right\}$ from $x$ to $y$. Thus there is $z \in X$ such that $d(z, x)<\epsilon$ and $d\left(F_{k n}(z), y\right)<\epsilon$. Consequently, $F_{k n}\left(\mathrm{~B}\left(x, r_{1}\right)\right) \cap \mathrm{B}\left(y, r_{2}\right) \neq \emptyset$. It follows that $F_{k}$ is topological transitive.

Theorem 3.5. Let $(X, d)$ be a compact metric space. Let $F_{n}$ be chain transitive for $n \in \mathbb{N}$. If $F$ has a weak stable point, then $F$ does not have the shadowing property.

Proof. Let $\epsilon>0$ and $x \in X$ be a weak stable point of $F$. Let $U=\cup_{i=1}^{s} U_{i}$ be a finite open covering of $X$ with $\operatorname{diam}\left(U_{i}\right)<\frac{\epsilon}{6}$. Then there exist $0<\eta<\frac{\epsilon}{6}$ and $n_{1}, n_{2}, \cdots, n_{s} \in \mathbb{N}$ such that for $y \in \mathrm{B}(x, \eta), F_{n_{i}}(y) \in U_{i}$ for $i=1,2, \cdots, s$. Take $T=\max \left\{\left|n_{i}\right|: 1 \leq i \leq s\right\}$. Then

$$
d\left(F_{n}(y), F_{n_{i}}(y)\right)<\frac{\epsilon}{6}
$$

for $n \in \mathbb{N},-T \leq i \leq T$. If $F$ has the shadowing property, then there exists $0<\delta<\eta$ such that each $\delta$-pseudo-orbit of $F$ can be $\eta$-shadowed by some point $t \in X$. By Lemma 3.4, $F$ is topological transitive, there exists $k \in \mathbb{N}$ such that $F_{-k}\left(\mathrm{~B}\left(x, \frac{\delta}{2}\right)\right) \cap \mathrm{B}\left(x, \frac{\delta}{2}\right) \neq \emptyset$. Take $z \in F_{-k}\left(\mathrm{~B}\left(x, \frac{\delta}{2}\right)\right) \cap \mathrm{B}\left(x, \frac{\delta}{2}\right)$. Since $F_{k}$ is chain transitive, there exists a $\delta$-chain $\left\{y=y_{0}, y_{1}, \cdots, y_{m}=z\right\}$ from $y$ to 
$z$. Thus $\left\{y, f_{1}(y), \cdots, F_{k-1}(y), y_{1}, f_{1}\left(y_{1}\right), \cdots, F_{k-1}\left(y_{1}\right), y_{2}, \cdots, y_{m-1}, f_{1}\left(y_{m-1}\right), \cdot \cdot\right.$ $\left.\cdot, F_{k-1}\left(y_{1}\right), z\right\}$ is a $\delta$-chain of $F$, which can be $\eta$-shadowed by some point $t \in X$. It follows that

$$
d(t, y)<\eta, \quad d\left(F_{(m+l) k}(t), z\right)<\eta, \quad l=0,1, \cdots .
$$

Note that $F_{(m+l) k}(t) \in X=\cup_{i=1}^{s} U_{i}$, then $F_{(m+l) k}(t) \in U_{i}$ for some $i=1, \cdots, s$. However, $F_{n_{i}}(y) \in U_{i}$. Therefore,

$$
\begin{aligned}
d\left(F_{(m+l) k}(y), z\right) & \leq d\left(F_{(m+l) k}(y), F_{n_{i}}(y)\right)+d\left(F_{n_{i}}(y), F_{(m+l) k}(t)\right)+d\left(F_{(m+l) k}(t), z\right) \\
& <\frac{\epsilon}{6}+\frac{\epsilon}{6}+\frac{\epsilon}{6}=\frac{\epsilon}{2} .
\end{aligned}
$$

Then

$$
d\left(F_{(m+l) k}(y), x\right) \leq d\left(F_{(m+l) k}(y), z\right)+d(z, x)<\frac{\epsilon}{2}+\frac{\epsilon}{12}=\frac{7 \epsilon}{12} .
$$

Consequently, $\overline{o\left(y, F_{k}\right)}-B(x, \epsilon) \subset\left\{y, F_{k}(y), \cdots, F_{(m-1) k}(y)\right\}$ is a finite set. Thus $\overline{o\left(y, F_{k}\right)} \neq X$, there exist $y^{*} \in X$ and $\lambda>0$ such that $B\left(y^{*}, \lambda\right) \subset$ $X-\overline{o\left(y, F_{k}\right)}$.

On the other hand, since $x$ is a weak stable point of $F$, it is a weak stable point of $F_{k}$. Thus there exists $\xi>0$ such that if $d(x, y)<\xi$ then $d\left(F_{k n_{i}}(x), F_{k n_{i}}(y)\right)<\frac{\lambda}{6}$ for $-T \leq i \leq T$. Due the topological transitivity of $F_{k}$, there is a point $\omega \in X$ such that $\overline{o\left(\omega, F_{k}\right)}=X$. Hence there exist $m, j \in \mathbb{N}$ with $-T \leq m-j \leq T$ such that

$$
d\left(F_{k j}(\omega), x\right)<\xi, \quad d\left(F_{k m}(\omega), y^{*}\right)<\frac{\lambda}{6} .
$$

Therefore,

$$
\begin{aligned}
& d\left(F_{(m-j) k}(y), y^{*}\right) \leq d\left(F_{(m-j) k}(y), F_{(m-j) k}(x)\right)+d\left(F_{(m-j) k}(x), F_{(m k}(\omega)\right)+d\left(F_{(m k}(\omega), y^{*}\right) \\
& <\frac{\lambda}{6}+\frac{\lambda}{6}+\frac{\lambda}{6}<\lambda,
\end{aligned}
$$

which contradicts with $B\left(y^{*}, \lambda\right) \subset X-\overline{o\left(y, F_{k}\right)}$. This completes the proof.

Theorem 3.6. Let $(X, F)$ be a non-autonomous dynamical system and $A$ be a dense invariant subset of $X$. Then $F$ is weak stable if and only if $\left.F\right|_{A}$ is weak stable. 
Proof. It is obvious that the weak stability of $F$ implies the same one of $\left.F\right|_{A}$. Conversely, assume that $\left.F\right|_{A}$ is weak stable. Fix any $x^{*} \in X$. Due density of $A$ and uniform continuity of $F$, for $\epsilon>0$, there exists $\delta>0$ such that if $z \in A \cap \mathrm{N}_{\delta}\left(x^{*}\right)$ then $d\left(F_{n}\left(x^{*}\right), F_{n}(z)\right)<\frac{\epsilon}{3}$. Since $\left.F\right|_{A}$ is weak stable, there is $T \in \mathbb{N}$ such that $d\left(F_{n}\left(x^{*}\right), F_{i}\left(x^{*}\right)\right)<\frac{\epsilon}{3}$ for $i=-T, \cdots, T$.

Take any $y \in X$ with $d\left(x^{*}, y\right)<\delta$, thus

$$
\begin{aligned}
d\left(F_{n}(y), F_{i}(y)\right) & <d\left(F_{n}(y), F_{n}\left(x^{*}\right)\right)+d\left(F_{n}\left(x^{*}\right), F_{i}\left(x^{*}\right)\right)+d\left(F_{i}\left(x^{*}\right), F_{i}(y)\right) \\
& <\frac{\epsilon}{3}+\frac{\epsilon}{3}+\frac{\epsilon}{3}=\epsilon
\end{aligned}
$$

for $i=-T, \cdots, T$. Therefore $F$ is weak chain continuous. This completes the proof.

Theorem 3.7. Let $(X, F)$ be a non-autonomous dynamical system and $(\mathcal{K}(X), \bar{F})$ be its induced set-valued system. Then $F$ is weak stable if and only if $\bar{F}$ is weak stable.

Proof. Assume that $F$ is weak stable. To prove that $\bar{F}$ is weak stable, by Theorem 3.6, it suffices to show that the weak stability of $\bar{F}$ on $\mathcal{K}_{\mathcal{F}}(X)$, as $\mathcal{K}_{\mathcal{F}}(X)$ is dense in $\mathcal{K}(X)$. Take $A=\left\{x_{1}, x_{2}, \cdots, x_{k}\right\} \in \mathcal{K}_{\mathcal{F}}(X)$. Since $F$ is weak stable, for $\epsilon>0$, there exist $\delta_{j}>0$ and $T_{j} \in \mathbb{N}$ such that

$$
d\left(F_{n}\left(y_{j}\right), F_{i}\left(y_{j}\right)\right)<\epsilon
$$

for every $y_{j} \in X$ with $d\left(x_{j}, y_{j}\right)<\delta_{j}$, where $i=-T_{j}, \cdots, T_{j}$ and $j=1, \cdots, k$.

Set $\delta=\max \left\{\delta_{j}\right\}$ and $T=\max \left\{T_{j}\right\}$. Let $B=\left\{y_{1}, y_{2}, \cdots, y_{k}\right\}$. Then $B \in \mathcal{K}_{\mathcal{F}}(X)$ satisfies the following

$$
H_{d}(A, B)<\delta
$$

and

$$
H_{d}\left(\bar{F}_{n}(A), \bar{F}_{i}(B)\right)<\epsilon,
$$

for $i=-T, \cdots, T$. It follows that $\bar{F}$ is weak stable.

Conversely, fix any $x \in X$. Then $\{x\} \in \mathcal{K}_{\mathcal{F}}(X)$. To prove $F$ is weak stable, it is sufficient to observe that

$$
d(x, y)=H_{d}(\{x\},\{y\})
$$

and

$$
H_{d}\left(\bar{F}_{n}(\{y\}), \bar{F}_{i}(\{y\})\right)=d\left(F_{n}(y), F_{i}(y)\right)
$$

for every $y \in X$ with $d(x, y)<\delta$. This completes the proof. 


\section{Acknowledgement(s)}

This work was supported by the National Natural Science Foundation of China (NO. 11601051), China Scholarship Council Contract (NO. 201608505146), and Natural Science Foundation Project of Chongqing CSTC (No. cstc2014jcyjA00054).

\section{References}

\section{References}

[1] Jose S. Cánovas, Li-Yorke chaos in a class of nonautonomous discrete systems, Journal of Difference Equations and Applications, 17 (2011) pp. $479-486$.

[2] Y.Shi, G.Chen, Chaos of time-varying discrete dynamical systems, Journal of Difference Equations and Applications, 15 (2009) pp. 429-449.

[3] Q.L. Huang, Y.M. Shi, and L.J. Zhang, Sensitivity of non-autonomous discrete dynamical systems, Applied Mathematics Letters,39 (2015) pp. $31-34$.

[4] X.X. Wu, P.Y. Zhu, Chaos in a class of non-autonomous discrete systems, Applied Mathematics Letter, 26 (2013) pp. 431-436.

[5] J.Dvoráková, Chaos in nonautonomous discrete dynamical systems, Communications in Nonlinear Science and Numerical Simulation, 17 (2012) pp. 4649-4652.

[6] A. Khan, P. Kumar, Chaotic phenomena and nonautonomous dynamical system, Global Journal of Theoretical and Applied Mathematics Sciences, 3 (2013) pp. 31-39.

[7] S.Yu. Pilyugin, O.B. Plamenevskaya, Shadowing is generic, Topology and its Applications, 97 (1999) pp. 253-266.

[8] S. Yu. Pilyugin, Shadowing in Structurally Stable Flows, Journal of Differential Equations, 140 (1997) pp. 238-265.

[9] B. Honary, A. Zamani Bahabadi, Orbital shadowing property, Bull. Korean Math. Soc. 45 (2008) pp. 645-C650. 
[10] K. Sakai, Various shadowing properties for positively expansive maps, Topology and its Applications, 131 (2003) pp. 15-31.

[11] Mukta Garg, Ruchi Das, Relations of the almost average shadowing property with ergodicity and proximality, Chaos, Solitons and Fractals, 91 (2016) pp. 430-433.

[12] X.X. Wu, P. Oprocha, and G.R. Chen, On various definitions of shadowing with average error in tracing, Nonlinearity, 29 (2016) pp. 1942-1972.

[13] Y.X. Niu, The average-shadowing property and strong ergodicity, Journal of Mathematical Analysis and Applications, 376 (2011) pp. 528-534.

[14] L. Fernández, C. Good, Shadowing for induced maps of hyperspaces, Fundamenta Mathematicae, 235 (2016) pp. 277-286.

[15] N.C. Bernardes Jr., R.M. Vermersch, On the dynamics of induced maps on the space of probability measures, Transaction of the American Mathematical Society, 368 (2016) pp. 7703-7725.

[16] N.C. Bernardes Jr., A. Peris and F. Rodenas, Set-Valued Chaos in Linear Dynamics, 88 (2017) pp. 451-463.

[17] J. Li, K. Yan and X. Ye, Recurrence properties and disjointness on the induced spaces, Discrete and Continuous Dynamical Systems, 45 (2015), pp. 1059-1073. 